Weckx, S.; D'Hulst, R.; Driesen, J., "Voltage Sensitivity Analysis of a Laboratory Distribution Grid With Incomplete Data," Smart Grid, IEEE Transactions on , vol.PP, no.99, pp.1,1

Digital Object Identifier: 10.1109/TSG.2014.2380642

URL: http://ieeexplore.ieee.org/stamp/stamp.jsp?tp=\&arnumber=6999971\&isnumber=5446437

IEEE. Personal use of this material is permitted. Permission from IEEE must be obtained for all other users, including reprinting/ republishing this material for advertising or promotional purposes, creating new collective works for resale or redistribution to servers or lists, or reuse of any copyrighted components of this work in other works. 


\title{
Voltage Sensitivity Analysis of a Laboratory Distribution Grid with Incomplete Data
}

\author{
Sam Weckx, Student Member, IEEE, Reinhilde D'Hulst, Johan Driesen, Senior Member, IEEE
}

\begin{abstract}
New voltage control algorithms are necessary to cope with the increasing amount of distributed generation and electric vehicles in distribution networks. Many of the newly proposed voltage control algorithms are based on linearised dependencies between the voltage magnitude and the active and reactive power consumption. These linearised dependencies are normally obtained by algorithms which rely on accurate grid topology information. Due to the traditionally passive operation of low voltage distribution networks, this information is typically missing, incomplete or inaccurate. Therefore, this work introduces a method to extract these linear dependencies based on historical smart meter data only. No information about the grid topology is required. The model adapts to the changing load conditions in the network. The algorithm has a low complexity and is applied to an unbalanced low voltage distribution network. Data of a practical laboratory setup is used to validate the proposed method in real-life conditions. With the obtained voltage sensitivity factors a voltage management strategy was implemented for the laboratory grid.
\end{abstract}

Index Terms-Grid identification, load flow, sensitivity analysis, smart metering, voltage control

\section{INTRODUCTION}

A high penetration of distributed generators and electric vehicles in Low Voltage (LV) distribution network challenges the future grid operation. More intelligent methods should be used for a better utilization of the distribution network, in order to maintain, or even to improve, the power-supply reliability and quality. Voltage rise is normally the main limiting factor to prevent the increase of photovoltaic (PV) generation in LV networks [1], while a high penetration of electric vehicles (EVs) can result in significant voltage drops. Therefore, Distribution System Operators (DSOs) are discovering a need to develop and validate new voltage management strategies [2].

The development of voltage management strategies is a challenging task due to the non-linear relationship between the network load and the grid voltage. Many of the developed voltage management strategies use a linearised model that describes the dependency of the voltage magnitude as a function of the power injections [3]-[23]. These linearised models are usually based on a voltage sensitivity analysis.

Sensitivity analysis at the transmission level is a routine task, where the sensitivity factors are typically obtained from the Jacobian matrix derived from the Newton Raphson load

S. Weckx is with ELECTA, KU Leuven, Kasteelpark Arenberg 10, bus 2445, 3001 Leuven, Belgium and with VITO, Boerentang 200 Mol, Belgium. $\mathrm{He}$ has a PhD fellowship of the Research Foundation Flanders (FWO-VITO). J. Driesen is with ELECTA, KU Leuven, Kasteelpark Arenberg 10, bus 2445, 3001 Leuven, Belgium.

R. D'Hulst is with VITO,Boerentang 200 Mol, Belgium. VITO and ELECTA are members of the Energyville research institute.

Corresponding author's email address: sam.weckx@esat.kuleuven.be flow method [24]. However, obtaining the Jacobian matrix for distribution networks is less practical, as load flow calculations need to be performed for every change in operating conditions. The relatively high $\mathrm{R} / \mathrm{X}$ ratio of $\mathrm{LV}$ distribution grids makes the Newton Raphson load flow method less suitable for solving the load flow problem [25]. A specific method to calculate the grid voltages in distribution grids is the backward-forward sweep method [26], which makes no use of the Jacobian matrix.

A popular alternative to obtain the voltage sensitivity factors is the 'perturb-and-observe' method, which consists of making small modifications and measuring the impact on the voltage magnitude [2]. This method is significantly less efficient due to the need to recompute the entire network state for a change on each bus. In order to increase the computational efficiency of the voltage sensitivity calculation in distribution grids of the previously mentioned methods, different alternatives have been proposed [24], [25], [27]-[30]. These methods still require information about grid topology and cable parameters to obtain the voltage sensitivity coefficients.

LV grids are the most unknown and manually controlled elements of the electricity grid [31]. Grid topology information is often not available in an easily accessible digital structure. The information that utilities have about the LV electricity grid is usually limited to which meter is connected to which LV substation [31]. Exact lengths between houses, or of the connecting cable between smart meter and distribution feeder are often not available. Moreover, the phase to which each household with a single phase connection is connected is often unknown [32]-[34]. This information is crucial for the previously mentioned algorithms. To overcome these problems, a computational intensive grid topology identification method has been developed which can only be applied under several assumptions [35]. However for most voltage management strategies, having the voltage sensitivity coefficients or a linear power flow model is sufficient.

In this paper an algorithm with low complexity is proposed that can obtain the voltage sensitivity factors and a linear load flow model based on historical smart meter data. No information about the grid topology is required. The proposed algorithm assumes that at every customer both active and reactive power are measured by a smart meter. The voltage magnitudes of nodes that are interesting for a DSO are measured by the smart meters. These nodes will be called the control nodes. The DSO can access this data which might be stored at a database. The low voltage network considered is an unbalanced three-phase four-wire radial system. Only radial operated distribution networks are considered, as these are the strict majority for the connection of residential customers to 
the grid. The network topology is considered to be fixed. In case the network gets reconfigured, new data will have to be collected to obtain the voltage sensitivity factors.

In the first part of this paper the algorithm is tested on an unbalanced distribution grid simulated in Matlab. In the last part actual data of a small laboratory grid is used to validate the algorithm in real-life conditions. This paper is structured as follows: the distribution grid used in the simulation results is described in Section II. In Section III, the algorithm that extracts the voltage sensitivity information from historical smart meter data is presented. It will be shown that the phase of connection of a single-phase household can be extracted from the sign of these voltage sensitivity factors. The obtained linear voltage model will be compared to the exact results in Section IV. It will be shown that the obtained linear voltage model makes errors of the same size as the non-linear system of load flow equations when there are realistic uncertainties on the applied load and grid models. Finally Section V discusses the practical laboratory grid where the algorithm is tested. The obtained voltage sensitivity factors of the laboratory grid are used to implement a voltage management strategy.

\section{Simulated NETWORK}

The network used in the simulations is an existing threephase four-wire radial distribution system with a TT earthing arrangement in Belgium. The network consists of 62 customers and is depicted in Fig. 1. This network is a semi-urban reference network in the LINEAR project [36].

All main feeder cables are of type EAXVB $1 \mathrm{kV}$ $4 \times 150 \mathrm{~mm}^{2}$ except for the cable between node A and node B, which is of type EAXVB $1 \mathrm{kV} 4 \times 95 \mathrm{~mm}^{2}$. Cable properties are defined in table I. The impedance values are calculated according to design specifications in the Belgian standard for underground distribution cables NBN C33-322 [37] with an assumed operating temperature of $45^{\circ} \mathrm{C}$. All households have a single-phase connection with a nominal line-to-neutral voltage of $230 \mathrm{~V}$ and are equally spread across the three phases. Statistically representative residential load profiles are available to perform load flow simulations. Generation of these load profiles is described in [38]. A constant power load model was assumed for $80 \%$ of this load, while a constant impedance load model was assumed for $20 \%$ of this load. This division between different load models generally varies in time for individual consumers [39], but in this work these are kept constant. All households with an odd number are equipped with a PV-installation with a nominal power of $3 \mathrm{~kW}$. The $\mathrm{PV}$ is modelled as a constant power load. The voltage at the secondary side of the transformer is considered to be $230 \mathrm{~V}$ during no load.

TABLE I

PROPERTIES OF THE SIMULATED NETWORK

\begin{tabular}{l|r|l} 
Properties & Value & Unit \\
\hline \hline Total feeder length & 1657 & {$[\mathrm{~m}]$} \\
Impedance of EAXVB $1 \mathrm{kV} 4 \times 95 \mathrm{~mm}^{2}$ & $0.352+0.078 \mathrm{j}$ & {$[\Omega / \mathrm{km}]$} \\
Impedance of EAXVB $1 \mathrm{kV} 4 \times 150 \mathrm{~mm}^{2}$ & $0.227+0.078 \mathrm{j}$ & {$[\Omega / \mathrm{km}]$}
\end{tabular}

\section{GRID IDENTIFICATION}

If distribution grid parameters, load profiles and load models are available, the voltages in all of the nodes of the grid can be calculated with a load flow algorithm. A non-linear set of equations describes the relation between the voltages in the grid and the power profiles:

$$
V_{h, t}=f\left(V_{t}^{0}, \mathbf{P}_{t}, \mathbf{Q}_{t}\right)
$$

where

- $V_{h, t}$ is the voltage magnitude of customer $h$ at timestep $t$

- $V_{t}^{0}$ is the voltage magnitude at the distribution transformer at timestep $t$

- $\mathbf{P}_{t}$ is a vector with the active power consumption of all customers at timestep $t$

- $\mathbf{Q}_{t}$ is a vector with the reactive power consumption of all customers at timestep $t$

For radial distribution networks, the backward-forward sweep method can be applied [26] to solve this non-linear system of equations that describes the relation between the complex powers of all customers and the voltage magnitude. The nonlinear load flow models are difficult to handle in optimization formulations due to their non-convexity. Besides that, the effect of changes in one part of the network on other parts of the network cannot be easily quantified due to the complexity of the model. Therefore the non-linear load flow models are sometimes approximated by a linear model, which can depend on the network loading [2], [7].

\section{A. A constant linear voltage model}

A first simple approximation consists of assuming a constant linear model:

$$
V_{h, t} \approx V_{t}^{0}+\sum_{\tilde{h}=1}^{N} a_{h, \tilde{h}} P_{\tilde{h}, t}+\sum_{\tilde{h}=1}^{N} b_{h, \tilde{h}} Q_{\tilde{h}, t},
$$

where

- $P_{\tilde{h}, t}$ is the active power of customer $\tilde{h}$ at timestep $t$

- $Q_{\tilde{h}, t}$ is the reactive power of customer $\tilde{h}$ at timestep $t$

- $a_{h, \tilde{h}}$ is the influence of active power of customer $\tilde{h}$ on the voltage magnitude of house $h$

- $b_{h, \tilde{h}}$ is the influence of reactive power of customer $\tilde{h}$ on the voltage magnitude of house $h$

The parameters of interest of this model, $a_{h, \tilde{h}}$ and $b_{h, \tilde{h}}$, can be obtained based on historical smart meter data. We assume that smart meters measure active and reactive power, as well as the voltage magnitude. In case a house has a three-phase connection to the grid, voltage and power are measured in each phase separately. Also the voltage magnitude at the distribution transformer is assumed to be measured. This data is stored in a database. If multiple time steps are available, a least squares problem (LS) can be recognised in (2) with $a_{h, \tilde{h}}$ and $b_{h, \tilde{h}}$ as unknowns. If the influence on the voltage of house $h$ needs to be defined, one can solve the following problem:

$$
\min _{a, b} \sum_{t=1}^{n_{t}}\left\|V_{h, t}-V_{t}^{0}-\sum_{\tilde{h}=1}^{N} a_{h, \tilde{h}} P_{\tilde{h}, t}-\sum_{\tilde{h}=1}^{N} b_{h, \tilde{h}} Q_{\tilde{h}, t}\right\|_{2}^{2},
$$




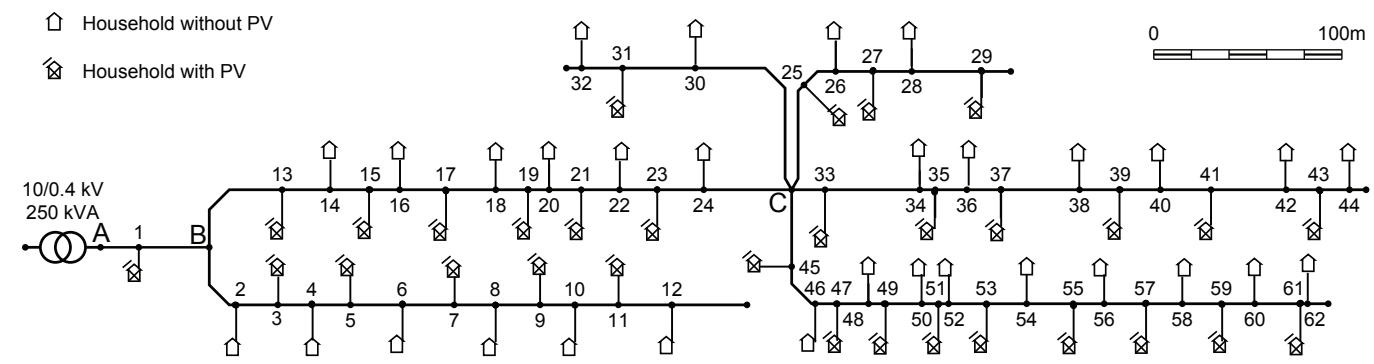

Fig. 1. The network used in the simulations. All lengths are drawn to scale.

where $n_{t}$ is the total amount of time steps available for least squares.

This linear least squares problem does not require any information about the grid. Nor the location or the order of the customers matters. Historic data is used to define the approximated voltage sensitivity factors $a_{h, \tilde{h}}$ and $b_{h, \tilde{h}}$. It is assumed that there is no electricity theft in the LV grid. This can be verified by comparing the sum of the measured power of all smart meters with the measurements at the substation transformer. If the measurements are sensitive to outliers, the 1 -norm can be applied in (3) instead of the 2-norm. When prior knowledge of the grid is available, this can be included by adding constraints to the LS problem (3) and making it a convex optimization problem [40] which can be efficiently solved [41].

An advantage is that the approximated voltage sensitivity factors are calculated off-line. Calculating the actual sensitivity factors in real-time is often too computationally intensive and requires a real-time communication of the load profiles of the different customers. Therefore some applications use already approximated sensitivity factors based on off-line load flow calculations that are sometimes updated depending on the network operation, e.g., heavy or light loading conditions [10], [11], [18]. By regularly updating the voltage control signals, small errors made by using approximated sensitivity factors can also be compensated [10].

\section{B. A piecewise linear voltage model}

In (2) the approximated sensitivity factors $a_{h, \tilde{h}}$ and $b_{h, \tilde{h}}$ are assumed to be constant. However, in [24] is shown that the actual sensitivity factors vary depending on the loading of the network. The approximated sensitivity factors $a_{h, \tilde{h}}$ and $b_{h, \tilde{h}}$ can also be made dependent on the network operation. The different captured time steps of the historical smart meter data are divided into different categories, e.g., heavy loading time periods, light loading periods and reverse power flow periods due to distributed generation. These categories can be found by summing up all the load profiles. The least squares problem (2) can then be solved for only those time steps of each category, e.g. for the low loading period $T^{\text {Low }}$ :

$$
\min _{a, b} \sum_{t \in T^{\text {Low }}}\left\|V_{h, t}-V_{t}^{0}-\sum_{\tilde{h}=1}^{N} a_{h, \tilde{h}} P_{\tilde{h}, t}-\sum_{\tilde{h}=1}^{N} b_{h, \tilde{h}} Q_{\tilde{h}, t}\right\|_{2}^{2},
$$

The solution of this least squares problem provides a set of approximated sensitivity factors for different levels of network loading. This model will therefore be called the piecewise linear model, whereas the one with the constant sensitivity factors will be called the constant linear model. The historical load and voltage profiles on which the model is based will be called the training data.

With the trained linear load flow model, the voltages at the customers nodes can be computed by evaluating (2) without any knowledge of the cable lengths. An important task for DSOs is to evaluate the impact on the grid voltage with an increasing amount of PV generation or an increasing usage of heat pumps and electric vehicles. The linear load flow model can be used to calculate the according voltage rise or drop when grid topology data is missing.

A disadvantage of the linear and piecewise linear load flow model is that it does not provide an estimate of the grid losses. It can only be used to define the voltage sensitivity factors, not the loss sensitivity factors. Therefore the main applications of these models is in voltage calculations and voltage control strategies of grids with missing or inaccurate grid data.

\section{Practical implementation}

To solve (4) the DSO needs to collect the load profiles of all households and the corresponding voltage profiles in which the DSO is interested. Smart meters can communicate their profiles to a database, where the DSO can access this data. Preferably the DSO will also check if there was any energy theft during this period.

By adding up the load profiles the system operator can divide the data into periods of different loading. The voltage sensitivity factors are now obtained by solving (4) for each period. Fig. 2 presents a flowchart of this procedure. Yearly (4) can be solved with the load and voltage profiles of the year before to check if the model still accurately describes the network behavior.

When the LV network gets reconfigured, (4) needs to be solved again with load and voltage profiles that occurred during the new reconfiguration. However, reconfiguration rarely occurs in European LV networks.

In Section IV the distribution grid of Fig. 1 is simulated and the load profiles and voltage profiles are obtained from this simulation environment. In Section V actual grid data of a laboratory distribution grid is accessed from a database and is used to calculate the voltage sensitivity factors. 


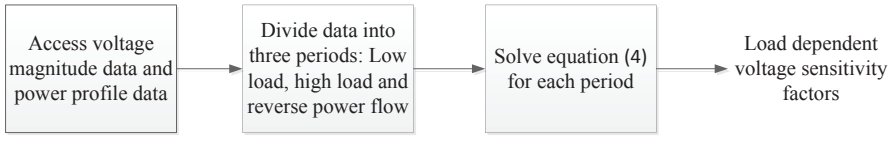

Fig. 2. Flowchart to obtain the load dependent voltage sensitivity factors based on historical smart meter data.

\section{Simulation Results}

The algorithm was tested on the network of Fig. 1. A piecewise linear model was calculated based on historic data of the previous month. This model was used to obtain the voltages in phase $\mathrm{V}$ of node 62 of a new day of which the measured load profiles were available. Fig. 3 compares the voltage obtained by an exact solution of the non-linear system of equations and the voltage obtained by the trained linear model. The non-linear system of equations was solved by the backward-forward sweep method [26]. Both the backwardforward sweep method and the training of the piecewise linear model was implemented in Matlab [42]. The errors by the piecewise linear model are clearly small.

In a second simulation the grid voltages for one month will be computed based on the available load profiles. An exact load flow with perfect knowledge of all cable parameters and load models does not make any errors when computing the grid voltages. However accurate grid or load data is rare. Different models can be made, with realistic errors on the cable lengths or load models. These models can be compared with the exact load flow model and with the trained models based on historical data. Four different load flow models will be compared to the exact load flow model.:

1) Inaccurate cable length: This load flow model consists of the non-linear load flow equations (1) of the grid in Fig. 1 but assumes a cable between node A and B which is $10 \%$ longer than the actual length. Therefore, the solution of this set of non-linear equations will make errors compared to the exact load flow model.
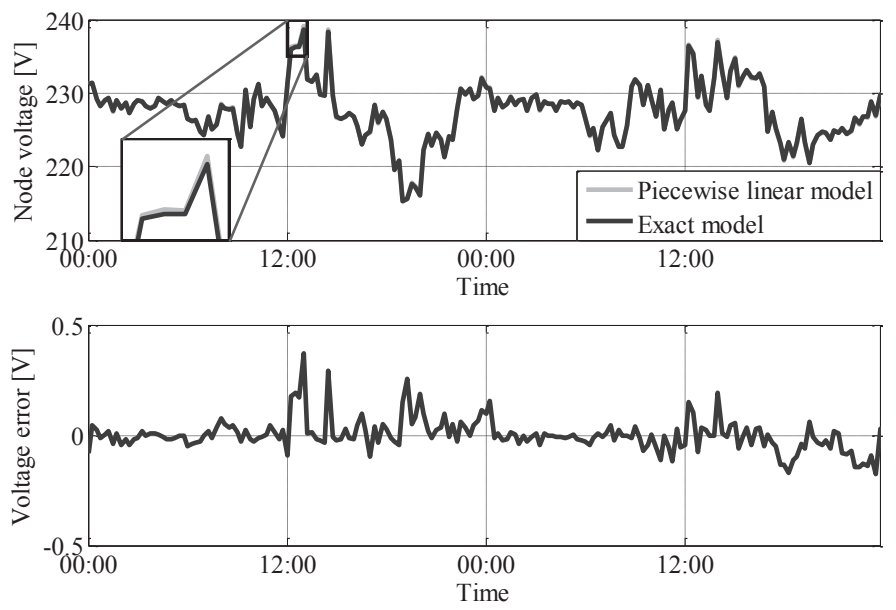

Fig. 3. At the top the voltage of node 62 for the exact load flow and by a trained piecewise linear model. At the bottom the error of the piecewise linear model compared to the exact load flow.
2) Inaccurate load models: The second load flow model consists of the non-linear load flow equations (1) of the grid in Fig. 1. However, the load models are assumed to be $100 \%$ constant power models, whereas the actual load models were chosen to be a power load model for $80 \%$ of this load and a constant impedance load model for $20 \%$ of the load. Therefore, the solution of this set of non-linear equations will make errors compared to the exact load flow model.

3) Constant linear model: The third load flow model is obtained by solving (3) based on historical load and voltage profiles of the previous month. No data about the grid topology or of the phase of connection of each customer was needed. This model will make errors due to the linearisation compared to the exact load flow model.

4) Piecewise linear model: The fourth load flow model is obtained by solving (4) based on historical load and voltage profiles of the previous month. No data about the grid topology or of the phase of connection of each customer was needed. This model will make errors due to the linearisation compared to the exact load flow model. This model can adapt to different loading conditions in the network.

Fig. 4 plots the probability density function (PDF) of the voltage error compared to the exact model of three representative nodes of the network for the four load flow models that were previously described. One of the nodes is located at the beginning of the feeder, one in the middle and one at the end. It can be seen that the errors are similar and that they slightly increase for nodes located at the end of the feeder. Note that these errors are small and will be in the range of the measurement noise. Using another grid and other load profiles will result in different errors made by the different load flow models. Statistically representative residential load profiles were used and the grid is an existing Belgian distribution grid.

Table II shows the Root Mean Square voltage Error (RMSE) and the Maximum Absolute voltage Error (MaxAE) of the four load flow models. It can be concluded from Fig. 4 and table II that the models that were trained based on historical data make small errors, but these are in the same range as when realistic errors are included in cable lengths or load models. Moreover, when the trained piecewise linear model is applied, errors can even be slightly smaller than in case of a load flow model with inaccurate grid or load data. So even with small realistic uncertainties on the cable lengths or load models, the trained linear or piecewise linear models become valid alternatives to the set of non-linear equations (1). Note that when there is no information about the phase of connection of each household, an exact solution of the non-linear system of equations can easily make errors of several Volts, especially when the PV generation is unequally distributed across the three phases.

The approximated sensitivities obtained by solving the least squares problem can be compared with the actual sensitivities. As was discussed earlier, the actual sensitivity factors vary depending on the loading of the network. In Fig. 5 the actual sensitivity factors are compared to the approximated sensitivities, where the influence of consumption of node 55 on the phase voltages of node 62 is presented. The actual sensitivity factors are calculated by the 'perturb-and-observe' method [2]. All households had an equal load, which ranged 

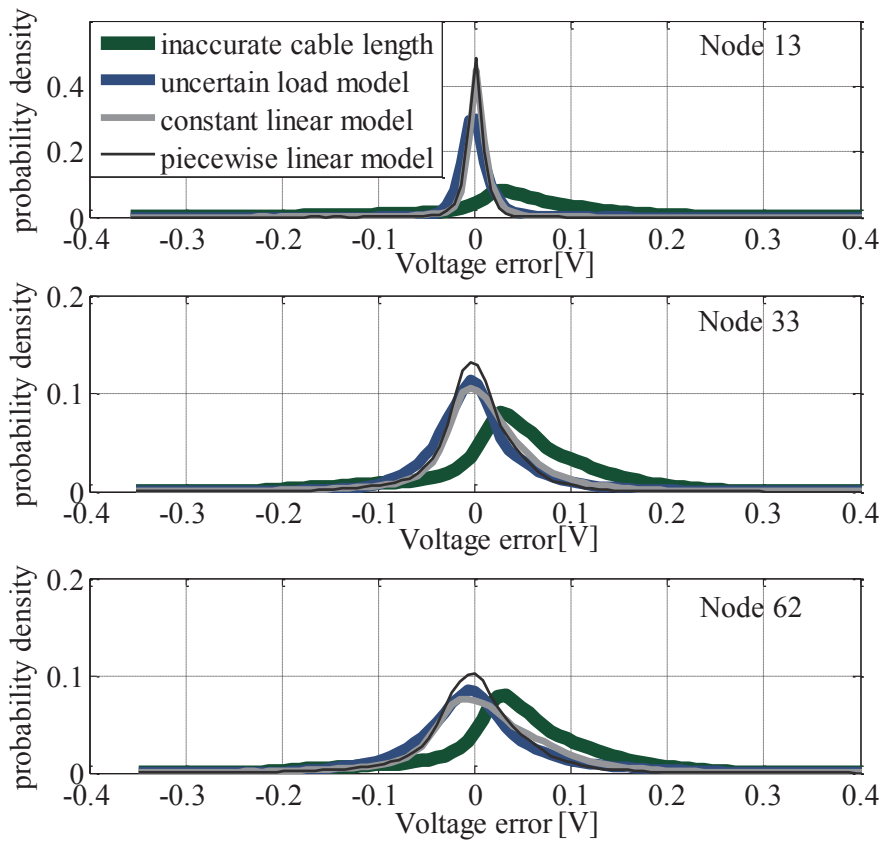

Fig. 4. Probability density function of the voltage error in three nodes for four different load flow models: 1) An exact load flow with inaccurate cable length information 2) An exact load flow with inaccurate assumptions regarding load models 3) A trained constant linear model 4) A trained piecewise linear model.

TABLE II

ESTIMATION ERRORS OF DIFFERENT LOAD FLOW MODELS

\begin{tabular}{l|c|c} 
Load flow model & RMSE [V] & MaxAE [V] \\
\hline \hline $\begin{array}{l}\text { Non-linear model with } \\
\text { inaccurate cable length }\end{array}$ & 0.089 & 0.42 \\
\hline $\begin{array}{l}\text { Non-linear model with } \\
\text { inaccurate load models }\end{array}$ & 0.054 & 0.69 \\
\hline $\begin{array}{l}\text { Trained linear model } \\
\text { based on historical data }\end{array}$ & 0.06 & 0.94 \\
\hline $\begin{array}{l}\text { Trained piecewise linear model } \\
\text { based on historical data }\end{array}$ & 0.043 & 0.69
\end{tabular}

from $-1 \mathrm{~kW}$ up to $1.5 \mathrm{~kW}$ to obtain the voltage sensitivity factors for different grid loading levels. The piecewise linear model gives a better approximation of the actual sensitivity factor. The available historical data was divided into three parts, each with the same amount of time steps, to obtain the piecewise linear model. As can be seen in Fig. 5 increasing the consumption of node 55 will reduce the voltage in phase $\mathrm{U}$ at node 62 , whereas it will increase the voltages of phase $\mathrm{V}$ and $\mathrm{W}$. This is due to the neutral point shift [43], [44]. Due to the resistive and inductive voltage drop across the neutral conductor, a load or PV unit connected to one phase will affect the phase voltages of the other phases. PV generation in one phase will increase the voltage of that phase, whereas the other two phase voltages decrease as shown in Fig. 6. Therefore it can be concluded that the load in node 55 is connected to phase U.

Due to the neutral displacement, the constants $a_{h, \tilde{h}}$ and $b_{h, \tilde{h}}$ obtained by the least squares method will give a measure of the location throughout the grid and the phase to which the household is connected. If the smart meter that measured the
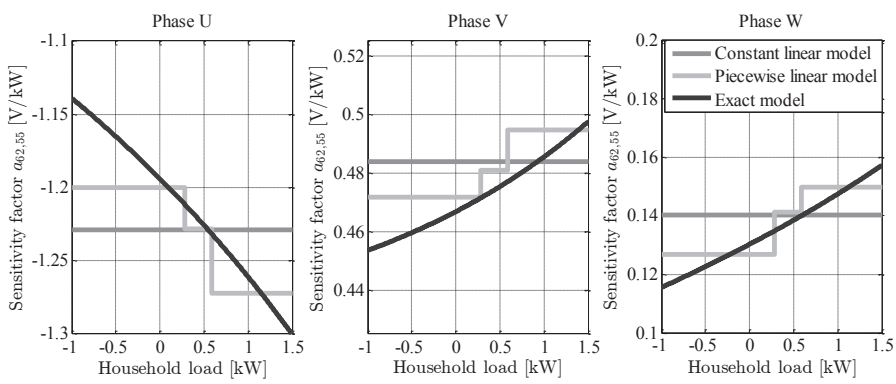

Fig. 5. The actual and the approximated voltage sensitivities of the phase voltages of node 62 due to active power consumption of household 55 for different grid loading levels.

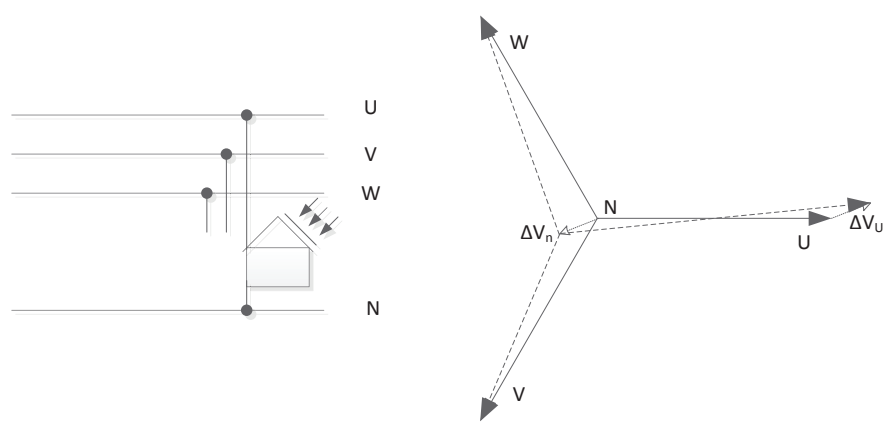

Fig. 6. The neutral displacement due to the injection of PV.

voltage $V^{h}$ is connected to the same phase as the household $\tilde{h}$, the constant $a_{h, \tilde{h}}$ will be negative, because the active power consumption has created a voltage drop. On the other hand, when the household is connected on another phase, $a_{h, \tilde{h}}$ will have a small positive value, due to the neutral point shift [43]. This allows all of the loads that are connected at the same phase to be grouped, based on the constants $a_{h, \tilde{h}}$ and $b_{h, \tilde{h}}$. Fig. 7 shows the influence of loads of all houses on the phase voltage of house 44 , when all households are connected by a single phase to the network. This is obtained by applying the least-squares method using historic data of one month. Obviously the consumption of node 44 has the biggest influence on the voltage of node 44 . All nodes with a negative sensitivity factor are connected to the same phase as household 44 . Node 42 and 43 are connected to another phase, and therefore extra consumption in these nodes will increase the voltage of household 44 . In this case there was an alternating sequence of $\mathrm{U}, \mathrm{V}, \mathrm{W}, \mathrm{U}, \mathrm{V}, \mathrm{W}, \ldots$ for the phase of connection of each household.

This method of phase identification is less computationally intensive as [33], where a mixed-integer program (MIP) is formed to identify the phase of connection of customers. [34] solves a combinatorial optimization problem by a Tabu search method. The phase of connection can also be identified by a unique signal injection, as proposed by [45]. This would require adapting the smart meter, which might be costly.

\section{Practical eValuation}

The methods described in section III are tested in the VITO HomeLab. The HomeLab is a Smart Grid research 


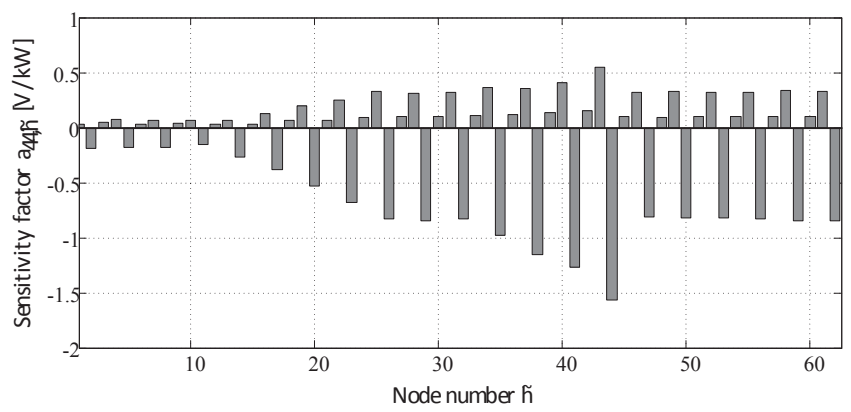

Fig. 7. The influence of active power of all houses on the phase voltage of house 44 .

and test facility for domestic energy management and demand response. The smart grid test infrastructure consists of roughly $300 \mathrm{~m}$ EAXVB $4 \times 16 \mathrm{~mm}^{2}$ cable to which different white good appliances can be connected as shown in Fig. 8. The consumption of four electric heaters can be managed by a solid state relay controlled by an Arduino unit. Five households can be simulated. The active and reactive power of each household are measured every $10 \mathrm{~s}$, as well as the phase voltage magnitude. The data is automatically saved in a SQL database.

Forming an exact load flow model that perfectly matches the measured voltages with the calculated voltages is a hard task, even for a small grid like this one. It requires several iterative adaptations of the non-linear system of equations, to cope with slight errors in cable parameters, cable lengths or load models. Moreover it is very difficult to identify the exact cause of the errors made by the exact model, which makes it a difficult task to adapt the correct parameters to improve the load flow
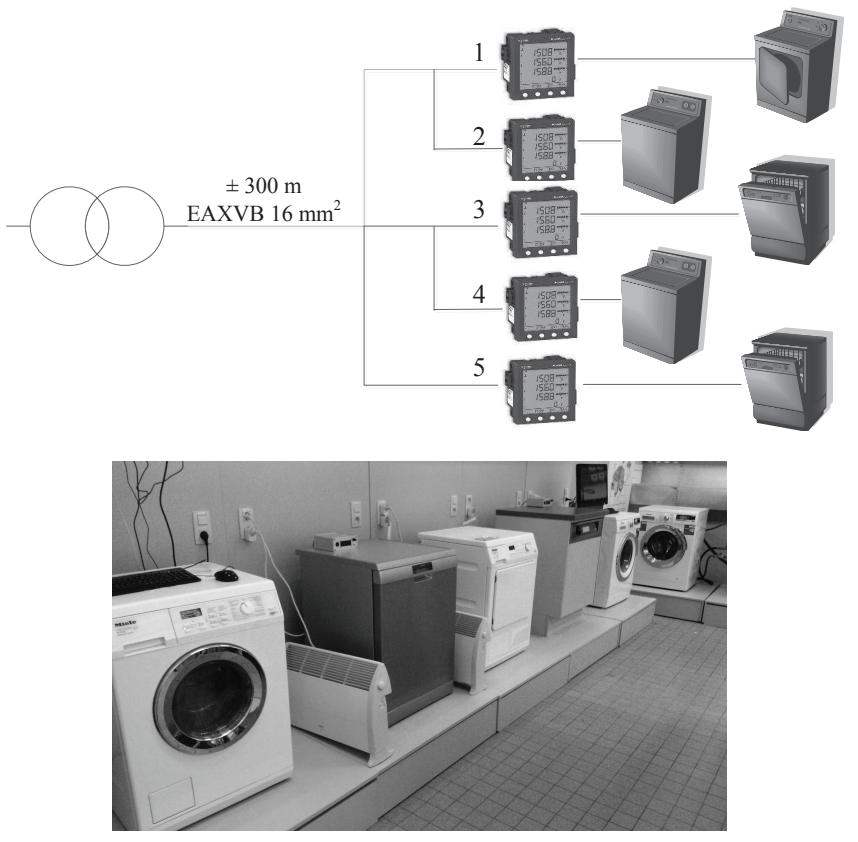

Fig. 8. The laboratory distribution network topology. model. Therefore the techniques described in Section III are applied to obtain a load flow model based on measured data, without knowledge of the grid data. Obviously, when an exact model is available this model should be preferred to obtain the voltage sensitivity factors.

\section{A. Grid identification}

During seven hours, different devices were turned on and off. The data of the first four hours was used as a training set to obtain the voltage sensitivity factors by solving (3). A constant linear model is trained. Due to the absence of distributed generation in the test grid, there are no significantly different operating points that require the use of the piecewise linear model of (4). The data of the last three hours is used as a validation set. Fig. 9 shows the measured voltage of this period in three nodes of the network and the voltages that are calculated based on the trained linear voltage model. The linear voltage model clearly resembles the measured voltages, without having any information on the grid topology, the load models or the phase of connection of each of the households. The errors made during this validation period are shown in Fig. 10 for each of the nodes in the network. For more than $95 \%$ of the time, the errors made by the constant linear model are less than $1 \mathrm{~V}$. The RMSE of this model equals $0.421 \mathrm{~V}$, while the MaxAE equals $2.06 \mathrm{~V}$.

The phase of connection of each household is also depicted in Fig. 10. The voltage sensitivity factors that express the influence of active power consumption on the voltages in node 1, 3 and 5 are presented in Fig. 11. It can be seen that nodes 1 and 2 are connected to the same phase, and nodes 3 and 4 are connected to another phase. When the phase of connection is extracted from the voltage sensitivity factors, the loading in each phase can be computed.

It can be concluded that a simple and effective constant linear load flow model can be computed based on measured
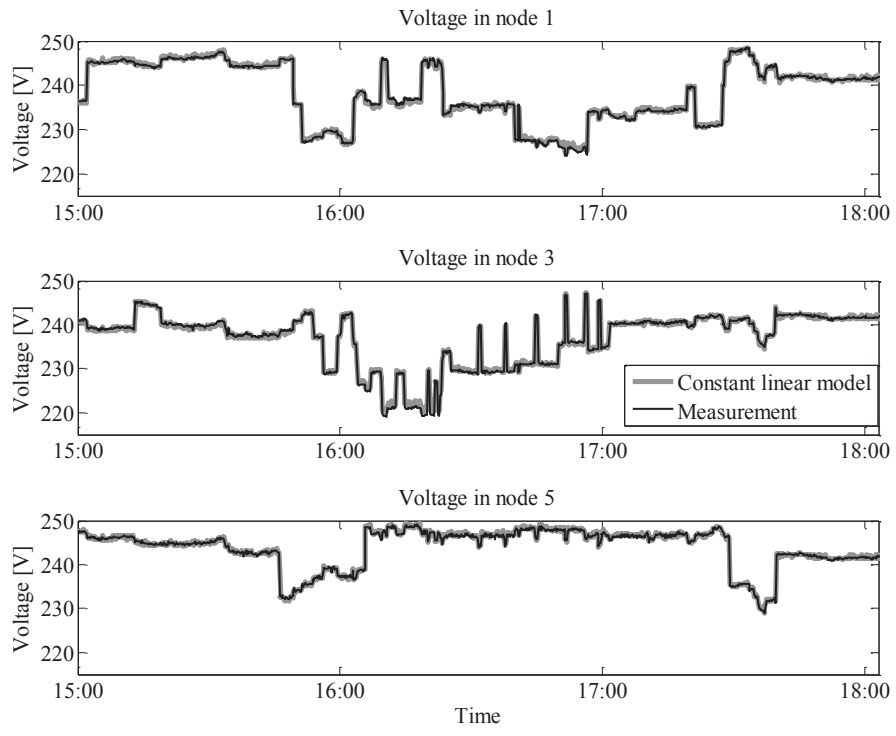

Fig. 9. The measured voltage and the voltage obtained by a linear model for three nodes of the test grid. 


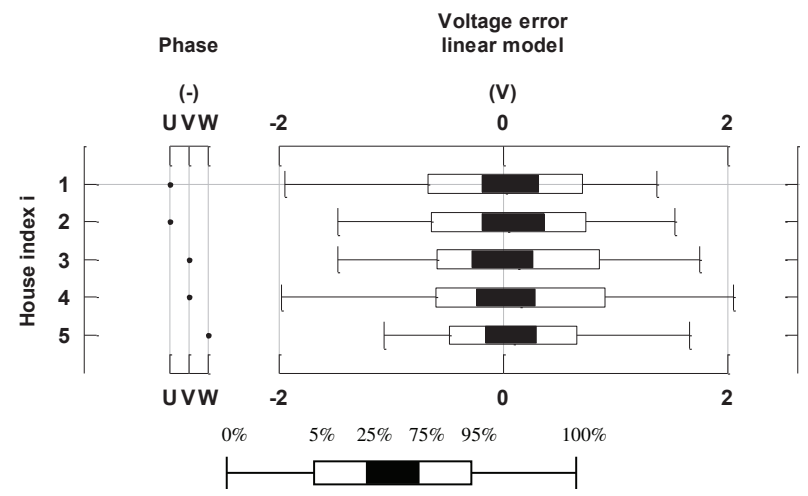

Fig. 10. The voltage estimation error by a constant linear model and the phase of connection of each of the loads.
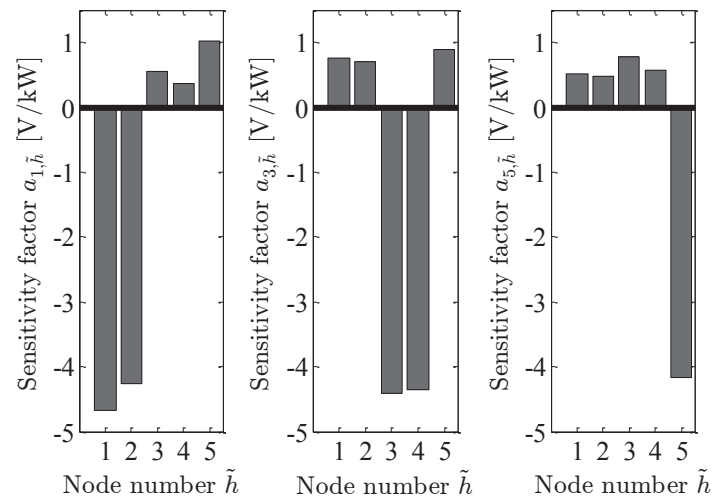

Fig. 11. The influence of active power consumption of all houses on the phase voltage of house 1 (left), house 3 (middle) and house 5 (right).

grid data. This linear model can be used in a voltage control strategy.

\section{B. Voltage control}

In the laboratory test grid voltage control strategies can be tested. These strategies can rely on the linear voltage model obtained in Section V-A. Here a voltage control strategy is proposed that makes use of one central control unit. This central unit receives from each EV a request to charge at a specified charging power. Each EV also defines the urgency of charging by one number, called the priority $\gamma$. Besides the information of the EVs the central unit also reads out the voltage of the nodes vulnerable to voltage problems.

With the sensitivity factors $a_{h, i}$ from the trained model, the control unit can calculate what the voltage would be if no EV was charging during the previous time step $\left(V_{h}^{n o E V}\right)$ :

$$
V_{h}^{n o E V} \approx V_{h, t-1}-\sum_{i=1}^{N_{E V s}} a_{h, i} P_{i}^{E V} x_{i, t}
$$

where

- $V_{h, t-1}$ is the measured voltage of node $h$

- $x_{i, t-1}$ binary variable that defines if $\mathrm{EV} i$ was charging during the previous timestep $t-1$

- $P_{i, t-1}^{E V}$ was the requested charging power by $\mathrm{EV} i$ at the previous timestep $t-1$
Now the central control unit can check if all EVs can charge at the same time without causing voltage problems. By making use of the sensitivity factors obtained in Section V-A, the expected voltage when all EVs would charge during the next timestep can be computed:

$$
V_{h, t} \approx V_{h}^{n o E V}+\sum_{i=1}^{N_{E V s}} a_{h, i} P_{i, t}^{E V}
$$

where

- $P_{i, t}^{E V}$ is the requested charging power by EV $i$ at timestep $t$

This expected voltage should be compared with the allowed voltage limits. The central control unit can give permission to all EVs to charge if this expected voltage is higher than the minimum allowed voltage. Otherwise, if all EVs can not charge all at the same time, the central unit will solve an optimization problem that tries to maximize the amount of EVs that can charge, weighted by their priority:

$$
\begin{array}{ll}
\max _{x_{i, t}, \varepsilon} & \sum_{i=1}^{N_{E V s}} \gamma_{i} x_{i, t}-\alpha \varepsilon \\
\text { subj. to } & x \in\{0,1\} \\
& V_{h, t}=V_{h}^{n o E V}+\sum_{i=1}^{N_{E V s}} a_{h, i} P_{i, t}^{E V} x_{i, t} \\
& V_{h, t} \geq V^{\text {min }}-\varepsilon \\
& \varepsilon \geq 0
\end{array}
$$

where

- $x_{i, t}$ binary variable that defines if EV $i$ can charge or not at timestep $t$

- $\varepsilon$ is a slack variable to keep the problem feasible

- $\alpha$ is a weighting factor of the slack variable

- $V^{\min }$ is the minimum voltage allowed in the grid

The priority of charging $\gamma$ should depend on the time left before departure and the required energy by the time of departure. EVs with a limited time before departure and still a high need for energy will have a high priority. Examples of how EVs can define their priority of charging can be found in [46]-[48]. The slack variable $\varepsilon$ is applied to guarantee a feasible problem [10]. Due to a high uncontrollable load it can become impossible to keep the voltage within limits at some moments. The slack variable keeps the problem feasible and makes sure that the algorithm tries to bring the voltage as close to the limits as possible if this occurs. The weighting factor of the slack variable is chosen ten times higher than the maximum possible priority.

The minimum voltage allowed in the grid $V^{\text {min }}$ depends on the applied voltage standard of the region of operation. In the laboratory setup, the minimum voltage is set at $227 \mathrm{~V}$ so that it will quickly go out of limits if the EV charging is not controlled.

The control signals $x_{i, t}$ can be send to all EVs when this optimization problem is solved. The control actions will give rise to a voltage which will be approximately equal to $V_{h}$. This procedure is shown in Fig. 12. The communication from the EVs to the central control unit can be made event based. 


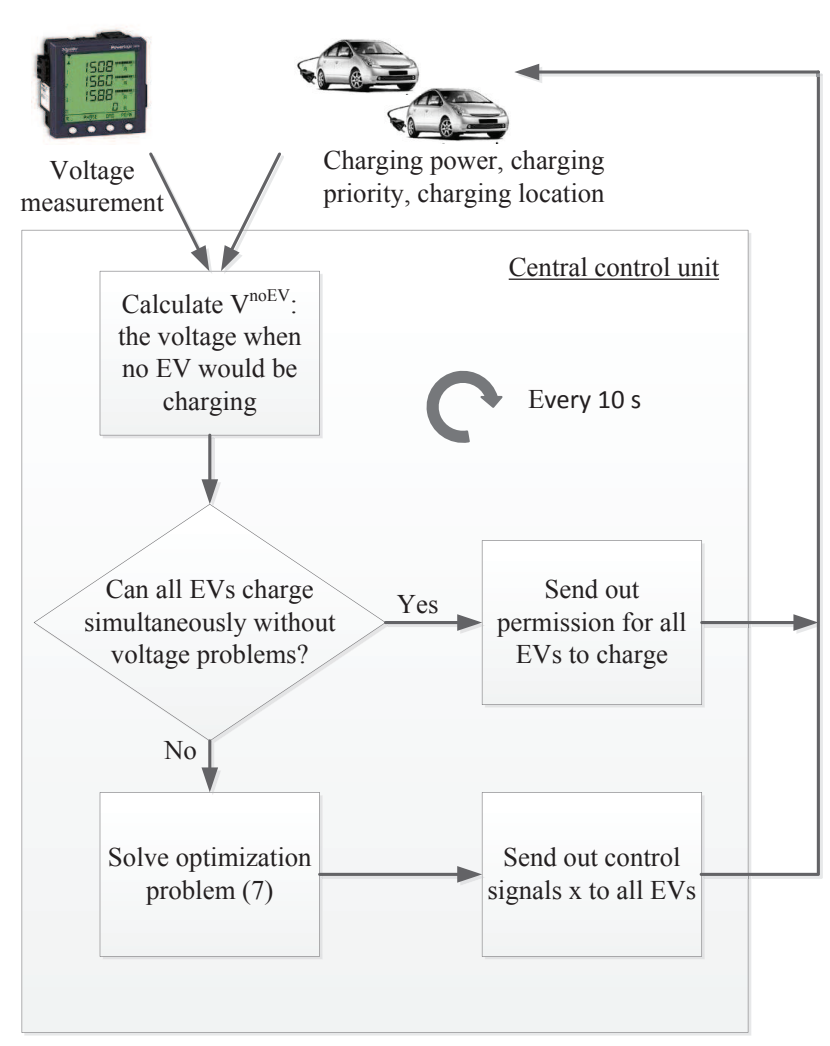

Fig. 12. Flowchart of the voltage control strategy for EVs executed by the central control unit.

Only when the EV adapts his priority or charging power he has to inform the central unit, otherwise the central unit can use the last sent values.

This voltage control strategy is tested in the VITO HomeLab. Fig. 13 presents the practical implementation of the central control unit. The central control unit is implemented in Matlab. The central unit communicates via a database with the Arduino load control units. Each Arduino control unit can switch a solid state relay to turn on or off an electric heater. The four available controllable electric heaters are used to emulate four EVs that charge at a rate of $1.6 \mathrm{~kW}$. One electric heater is connected to node 1 , one to node 2 , one to node 3 and one to node 4 .

It is assumed that the emulated EVs connected to node 1 and 3 have a high need for the requested energy, while the emulated EVs connected to node 2 and 4 have a low need for the requested energy. Therefore the priority $\gamma$ of EVs 1 and 3 is higher than the priority of EVs 2 and 4 . The different priorities are given in table III. For the ease of presentation, the priorities are kept constant during the test. The central control unit checks the network conditions every $10 \mathrm{~s}$ and solves optimization problem (7) if necessary.

The total load in each phase during this test is plotted in Fig. 14, while the voltage is shown in Fig. 15. It is clear from the plotted total load that the EVs can charge when the total load is low in their phase, and have to stop charging when the uncontrollable load by the households is high. When an EV charges and the uncontrollable load suddenly rises, the voltage

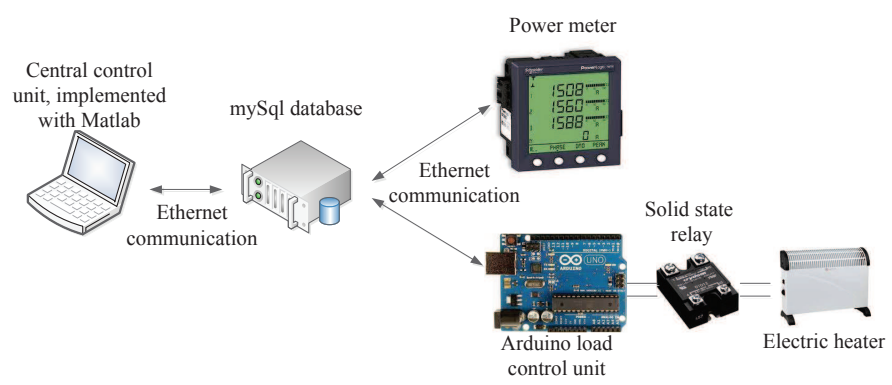

Fig. 13. Practical implementation of the voltage control strategy.

TABLE III

THE USED PRIORITIES OF THE FOUR EVS IN THE PRACTICAL SETUP

\begin{tabular}{c|c|c|c|c} 
& EV 1 & EV 2 & EV 3 & EV 4 \\
\hline Priority & 0.8 & 0.6 & 0.75 & 0.5
\end{tabular}

drops. The control unit quickly corrects the voltage drop by stopping the charging of the EV in this phase. I.e. at 12:30 it quickly stops the charging of the EVs connected to phase $\mathrm{V}$ after a sudden increase of the uncontrollable load. This way the voltage drops only for a short period of time below the acceptable limits.

Two EVs are connected to phase $\mathrm{U}$ and two to phase V. At some moments the total uncontrollable load is high in one phase, and only one of the two EVs connected to a phase can charge to comply with the voltage limits. In this case the optimization control problem lets the EV with the highest priority charge, while the other EV has to stop charging. In Fig. 14 it can be seen that at 13:00 EV 1 can charge, but EV 2 has to stop charging because otherwise the voltage will drop to an unacceptable level.

Problem (7) can be extended with constraints that limit the total load in each phase. Future work focusses on testing and validating different voltage management strategies with the obtained voltage sensitivity factors.

\section{CONCLUSION}

In this paper a linearised load flow model is identified by means of historical smart meter data for radial distribution grids with incomplete or inaccurate data. A linearised load flow model which depends on the loading of the network could be obtained by solving a least squares problem. No information concerning grid topology, load models or the phase of connection was required. It was shown that the voltage sensitivities obtained based on historical smart meter data are accurate approximations of the actual voltage sensitivities. The approximated voltage sensitivities provide information about both the location and the phase of each customer. These voltage sensitivities are crucial for many voltage management strategies.

The algorithm has been verified in a practical laboratory environment. A validation set of data was used to compare the output of a trained linearised load flow model with actual data, which were shown to match accurately. The algorithm could also correctly identify the phase to which each household was connected. As an example of the many applications, the 
Active power in phase $\mathrm{U}$
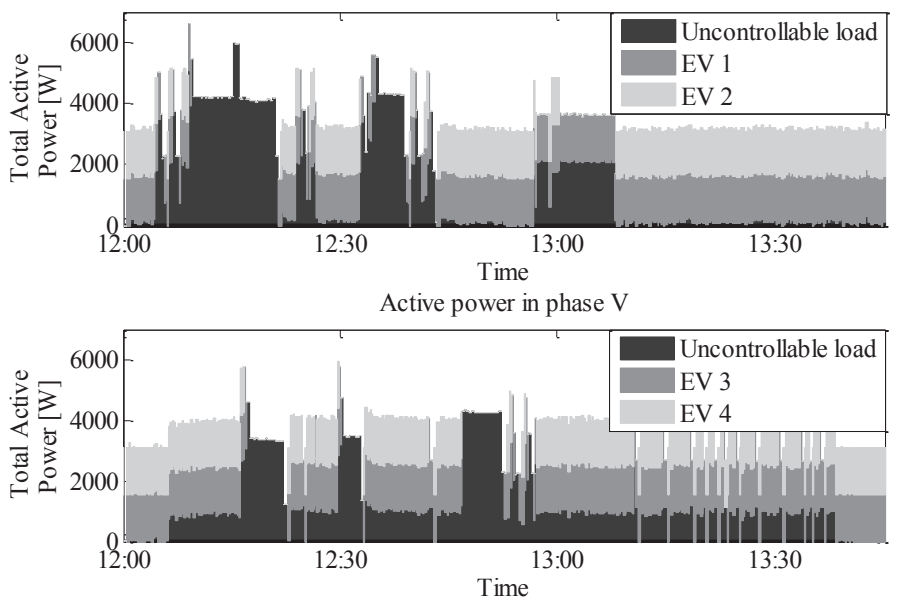

Active power in phase $\mathrm{W}$

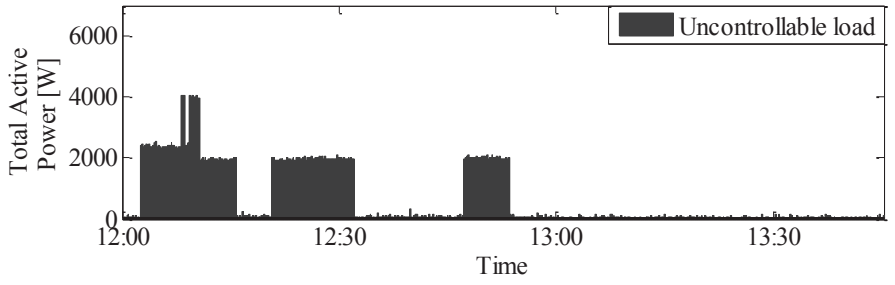

Fig. 14. The total active power consumption in phase $\mathrm{U}, \mathrm{V}$ and $\mathrm{W}$ during the test of the implemented voltage management strategy.
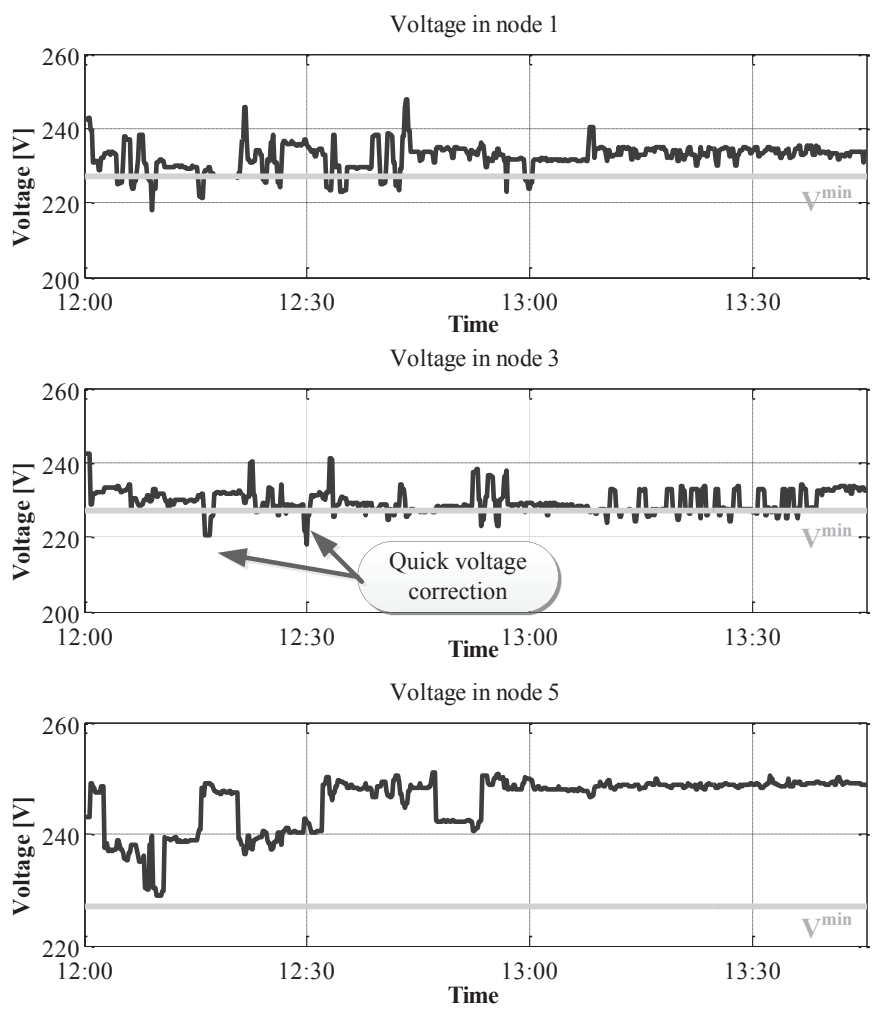

Fig. 15. The voltages of nodes connected to phase U (node 1), V (node 3 ) and $\mathrm{W}$ (node 5) during the test of the implemented voltage management strategy. voltage sensitivities were used in a voltage control strategy. A central control unit evaluates if the requested power of an EV can be consumed without harming the grid. When the voltage dropped below the allowed limits, the control unit could quickly correct the voltage to an acceptable level.

\section{ACKNOWLEDGEMENT}

The authors would like to thank Johan De Winter, Guy Meynen, Filip Leemans and Marcel Stevens for the guidance and technical support during the laboratory tests.

\section{REFERENCES}

[1] G. Mokhtari, A. Ghosh, G. Nourbakhsh, and G. Ledwich, "Smart robust resources control in lv network to deal with voltage rise issue," IEEE Trans. on Sustain. Energy, vol. 4, no. 4, pp. 1043-1050, Oct 2013.

[2] F. Tamp and P. Ciufo, "A sensitivity analysis toolkit for the simplification of mv distribution network voltage management," IEEE Trans. on Smart Grid, vol. 5, no. 2, pp. 559-568, March 2014.

[3] M. Baran and I. El-Markabi, "A multiagent-based dispatching scheme for distributed generators for voltage support on distribution feeders," IEEE Trans. Power Syst., vol. 22, no. 1, pp. 52-59, Feb 2007.

[4] G. Mokhtari, G. Nourbakhsh, and A. Ghosh, "Smart coordination of energy storage units (esus) for voltage and loading management in distribution networks," IEEE Trans. Power Syst., vol. 28, no. 4, pp. 4812-4820, Nov 2013.

[5] P. Richardson, D. Flynn, and A. Keane, "Optimal charging of electric vehicles in low-voltage distribution systems," IEEE Trans. Power Syst. vol. 27, no. 1, pp. 268-279, Feb 2012.

[6] M. Nick, R. Cherkaoui, and M. Paolone, "Optimal allocation of dispersed energy storage systems in active distribution networks for energy balance and grid support," IEEE Trans. Power Syst., vol. PP, no. 99, pp. 1-11, 2014.

[7] F. Marra, G. Yang, C. Traeholt, E. Larsen, J. Ostergaard, B. Blazic, and W. Deprez, "Ev charging facilities and their application in lv feeders with photovoltaics," IEEE Trans. Smart Grid, vol. 4, no. 3, pp. 15331540, Sept 2013.

[8] A. Keane and M. O’Malley, “Optimal allocation of embedded generation on distribution networks," IEEE Trans. Power Syst., vol. 20, no. 3, pp. 1640-1646, Aug 2005.

[9] D. Popovic, J. Greatbanks, M. Begovic, and A. Pregelj, "Placement of distributed generators and reclosers for distribution network security and reliability," Int. J. of Electrical Power and Energy Systems, vol. 27, no. 5?6, pp. 398 - 408, 2005.

[10] G. Valverde and T. Van Cutsem, "Model predictive control of voltages in active distribution networks," IEEE Trans. on Smart Grid, vol. 4, no. 4 pp. 2152-2161, Dec 2013.

[11] F. Milano and O. Hersent, "Optimal load management with inclusion of electric vehicles and distributed energy resources," IEEE Trans. on Smart Grid, vol. 5, no. 2, pp. 662-672, March 2014.

[12] L. Yu, D. Czarkowski, and F. de Leon, "Optimal distributed voltage regulation for secondary networks with dgs," IEEE Trans. on Smart Grid, vol. 3, no. 2, pp. 959-967, June 2012.

[13] P. Richardson, D. Flynn, and A. Keane, "Local versus centralized charging strategies for electric vehicles in low voltage distribution systems," IEEE Trans. on Smart Grid, vol. 3, no. 2, pp. 1020-1028, June 2012.

[14] S. Weckx, C. Gonzalez, and J. Driesen, "Combined central and local active and reactive power control of pv inverters," IEEE Trans. on Sust. Energy, vol. PP, no. 99, pp. 1-1, 2014.

[15] Q. Zhou and J. Bialek, "Generation curtailment to manage voltage constraints in distribution networks," Generation, Transmission Distribution, IET, vol. 1, no. 3, pp. 492-498, May 2007.

[16] A. Borghetti, M. Bosetti, S. Grillo, S. Massucco, C. Nucci, M. Paolone, and F. Silvestro, "Short-term scheduling and control of active distribution systems with high penetration of renewable resources," Systems Journal, IEEE, vol. 4, no. 3, pp. 313-322, Sept 2010.

[17] P. Pachanapan, O. Anaya-Lara, A. Dysko, and K. Lo, "Adaptive zone identification for voltage level control in distribution networks with dg," IEEE Trans. on Smart Grid, vol. 3, no. 4, pp. 1594-1602, Dec 2012. 
[18] R. Tonkoski, L. A. Lopes, and T. H. El-Fouly, "Coordinated active power curtailment of grid connected PV inverters for overvoltage prevention," IEEE Trans. Sustain. Energy, vol. 2, no. 2, pp. 139-147, 2011.

[19] S. Weckx, J. Driesen, and R. D'hulst, "Optimal real-time pricing for unbalanced distribution grids with network constraints," in Power and Energy Society General Meeting (PES), 2013 IEEE, July 2013, pp. 1-5.

[20] A. Samadi, R. Eriksson, L. Soder, B. Rawn, and J. Boemer, "Coordinated active power-dependent voltage regulation in distribution grids with pv systems," IEEE Trans. Power Del., vol. 29, no. 3, pp. 1454-1464, June 2014.

[21] M. A. Kashem and G. Ledwich, "Distributed generation as voltage support for single wire earth return systems," IEEE Trans. Power Del., vol. 19, no. 3, pp. 1002-1011, July 2004.

[22] B. Robbins, C. Hadjicostis, and A. Dominguez-Garcia, "A two-stage distributed architecture for voltage control in power distribution systems," IEEE Trans. Power Syst., vol. 28, no. 2, pp. 1470-1482, May 2013.

[23] V. Calderaro, G. Conio, V. Galdi, G. Massa, and A. Piccolo, "Optimal decentralized voltage control for distribution systems with inverter-based distributed generators," IEEE Trans. Power Syst., vol. 29, no. 1, pp. 230241, Jan 2014.

[24] Q. Zhou and J. Bialek, "Simplified calculation of voltage and loss sensitivity factors in distribution networks," in Proc. of the 16th Power Systems Computation Conference (PSCC2008), Glasgow, Scotland, 2008.

[25] D. Khatod, V. Pant, and J. Sharma, "A novel approach for sensitivity calculations in the radial distribution system," IEEE Trans. on Power Delivery, vol. 21, no. 4, pp. 2048-2057, Oct 2006.

[26] C. Cheng and D. Shirmohammadi, "A three-phase power flow method for real-time distribution system analysis," IEEE Trans. on Power Syst., vol. 10 , no. 2 , pp. $671-679$, may 1995 .

[27] K. Christakou, J. LeBoudec, M. Paolone, and D.-C. Tomozei, "Efficient computation of sensitivity coefficients of node voltages and line currents in unbalanced radial electrical distribution networks," IEEE Trans. on Smart Grid, vol. 4, no. 2, pp. 741-750, June 2013.

[28] I. Dzafic, R. Jabr, E. Halilovic, and B. Pal, "A sensitivity approach to model local voltage controllers in distribution networks," IEEE Trans. Power Syst., vol. 29, no. 3, pp. 1419-1428, May 2014.

[29] M. Brenna, E. De Berardinis, L. Delli Carpini, F. Foiadelli, P. Paulon, P. Petroni, G. Sapienza, G. Scrosati, and D. Zaninelli, "Automatic distributed voltage control algorithm in smart grids applications," IEEE Trans. on Smart Grid, vol. 4, no. 2, pp. 877-885, June 2013.

[30] J. Marti, H. Ahmadi, and L. Bashualdo, "Linear power-flow formulation based on a voltage-dependent load model," IEEE Trans. on Power Del., vol. 28, no. 3, pp. 1682-1690, July 2013.

[31] L. Marron, X. Osorio, A. Llano, A. Arzuaga, and A. Sendin, "Low voltage feeder identification for smart grids with standard narrowband plc smart meters," in Power Line Communications and Its Applications (ISPLC), 2013 17th IEEE International Symposium on, March 2013, pp. $120-125$.

[32] H. Pezeshki and P. Wolfs, "Consumer phase identification in a three phase unbalanced lv distribution network," in Innovative Smart Grid Technologies (ISGT Europe), 2012 3rd IEEE PES International Conference and Exhibition on, Oct 2012, pp. 1-7.

[33] V. Arya, D. Seetharam, S. Kalyanaraman, K. Dontas, C. Pavlovski, S. Hoy, and J. R. Kalagnanam, "Phase identification in smart grids," in Proc. IEEE Int Smart Grid Communications (SmartGridComm) Conf, 2011, pp. 25-30.

[34] M. Dilek, "Integrated design of electrical distribution systems: Phase balancing and phase prediction case studies," Ph.D. dissertation, Blacksburg Virginia, 2001.

[35] S. Bolognani and L. Schenato, "Identification of power distribution network topology via voltage correlation analysis," Proc. IEEE Conf. on Decision and Control, 2013.

[36] E. Peeters, C. Develder, J. Das, J. Driesen, and R. Belmans, "Linear: towards a breakthrough of smart grids in Flanders," in Proc. 2nd Int. Conf. Innovation for Sustainable Production, vol. 3, Bruges, Belgium, 18-21 Apr. 2010, pp. 3-6.

[37] Kabels Voor Ondergrondse Aanleg, met Synthetische Isolatie en Versterkte Mantel (Type $1 \mathrm{kV}$ ), NBN Std. C 33-322, 1975 Std.

[38] W. Labeeuw and G. Deconinck, "Residential electrical load model based on mixture model clustering and markov models," IEEE Trans. on Ind. Inf., vol. 9, no. 3, pp. 1561-1569, 2013.

[39] H. L. Willis, Power Distribution Planning Reference Book. Abingdon: Dekker, 2004.

[40] S. Weckx, C. Gonzalez, J. Tant, T. D. Rybel, and J. Driesen, "Parameter identification of unknown radial grids for theft detection." in ISGT Europe. IEEE, pp. 1-6.
[41] S. Boyd and L. Vandenberghe, Convex Optimization. Cambridge University Press, Mar. 2004

[42] MATLAB, (R2013a). The MathWorks Inc., 2013.

[43] L. Degroote, B. Renders, B. Meersman, and L. Vandevelde, "Neutralpoint shifting and voltage unbalance due to single-phase DG units in low voltage distribution networks," in Proc. IEEE Bucharest PowerTech, 2009, pp. 1-8.

[44] C. Gonzalez, J. Geuns, S. Weckx, T. Wijnhoven, P. Vingerhoets, T. De Rybel, and J. Driesen, "LV distribution network feeders in Belgium and power quality issues due to increasing PV penetration levels," in 3rd IEEE PES Innovative Smart Grid Technologies Conf., Berlin, Germany, October 14-17 2012.

[45] Caird, "Meter phase identification," US patent application Patent 20100164 473, 2010

[46] S. Weckx, R. D'Hulst, and J. Driesen, "Optimal frequency support by dynamic demand." in PowerTech, 2013 IEEE Grenoble, 2013, pp. 1-6.

[47] K. De Craemer and G. Deconinck, "Balancing trade-offs in coordinated phev charging with continuous market-based control," IEEE PES Innovative Smart Grid Technologies 2012, 2012.

[48] M. D. Galus, S. Koch, and G. Andersson, "Provision of load frequency control by phevs, controllable loads, and a cogeneration unit," IEEE Trans. on Ind. Electron.,, vol. 58, no. 10, pp. 4568-4582, 2011.

\section{BIOGRAPHIES}

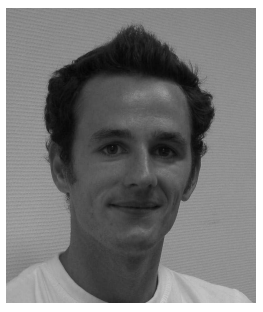

Sam Weckx (S'11) received the M.Sc. degree in Electrical Engineering in 2009 and Mechanical Engineering in 2010 from the Katholieke Universiteit Leuven (KU Leuven), Belgium, where he is currently working towards the Ph.D. degree as a research assistant with the division ESAT-ELECTA. His research interests include the application of distributed optimization in smart grids and voltage control in distribution networks.

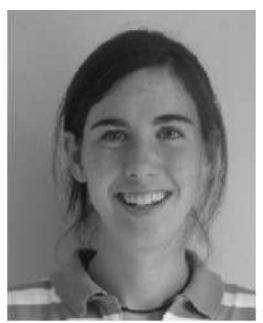

Reinhilde D'Hulst graduated in 2004 as M.Sc. in Electrical Engineering from the Katholieke Universiteit Leuven (KU Leuven). She obtained her Ph.D. in Electrical Engineering from the KU Leuven in 2009 after working on power management circuits for energy harvesters. Currently she works for VITO, the Flemish Institute for Technological Research where she is involved in several research projects related to Smart Grids. Currently she does research on grid-connecting issues of renewable energy resources and control algorithms for Demand Side

Management

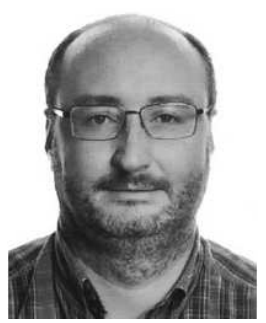

Johan Driesen (S'93 - M'97 - SM'12) received the M.Sc. and Ph.D. degrees in Electrical Engineering from the Katholieke Universiteit Leuven (KU Leuven), Leuven, Belgium, in 1996 and 2000 , respectively. Currently, he is a Professor with the KU Leuven and teaches power electronics and electric drives. In 2000, he was with the Imperial College of Science, Technology and Medicine, London, U.K. In 2002, he was with the University of California, Berkeley. Currently, he conducts research on distributed generation, power electronics, and its 